\title{
A case of eosinophilic cystitis in patients with abdominal pain, dysuria, genital skin hyperemia and slight toxocariasis
}

\author{
Maria Angela Cerruto, Carolina D'Elia, Walter Artibani \\ Urology Clinic, University of Verona, Italy.
}

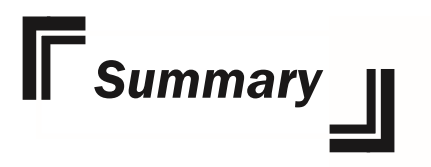

Eosinophilic cystitis is a rare inflammatory disease with controversial aetiology and treatment.

We report the case of a 61-year-old man presented with lower quadrant abdominal pain and lower urinary tract symptoms, non responsive to antibiotics and nonsteroidal antiinflammatory drugs.

Physical examination was substantially negative, such as laboratory parameters, microscopic, bacteriological and serological evaluations. Cystoscopy revealed red areas involving the mucosa of the bladder and transurethral biopsies revealed infiltrating eosinophils.

The patient was treated with corticosteroids and montelukast sodium with improving of the symptoms, and at 5 weeks postoperative pain score was reduced.

After discontinuing corticosteroids dysuria recurred with the development of hyperemia at the genital skin; the specific enzyme-linked immunosorbent assay (ELISA) to detect antibodies against several parasites was slightly positive for Toxocara species. Montelukast sodium was discontinued and corticosteroid therapy was started together with albendazole, with improving of patient's symptoms and pain decreasing after one week.

KEY WORDS: Eosinophilic cystitis; Dysuria; Genital skin hyperemia; Toxocariasis.

\section{INTRODUCTION}

Eosinophilic cystitis is a rare inflammatory disease, that may affect adults and children. The aetiology and the treatment of this condition remains controversial.

The pathological presentation of this disease is a eosinophilic infiltration, ranging from mild inflammation to bladder fibrosis. His clinical presentation includes lower urinary tract symptoms, thath can simulate an urinary tract infections.

\section{Case report}

A 61-year-old man presented to the emergency room with a 1-week history of constant lower quadrant abdominal pain and lower urinary tract symptoms (1) characterized by increased daytime frequency, slow stream, nocturia, bladder and urethral pain increasing with bladder filling and reducing during and after voiding, nonresponsive to antibiotics and nonsteroidal antiinflammatory drugs.

The patient's past medical history was notable for hypertension diagnosed 5 years earlier and well controlled with ramipril $5 \mathrm{mg}$ daily. No history of allergy was obtained. He denied having any domestic pets, high-risk sexual behaviour, the use of tobacco, alcohol or illicit drugs, had no sick contacts and hat not travelled recently. He denied having any visual changes or respiratory symptoms, chest pain, nausea, vomiting, melena or hematochezia. He reported changes in bowel habits (an increasing stipsis). Upon physical examination skin rash, upper quadrant abdominal tenderness or palpable liver, spleen or abdominal mass were all absent. Only a slight tenderness to palpation in the lower abdominal quadrants, mainly in the suprapubic 
region, was noted. Digital rectal examination revealed an indolent regular prostate. Urethral and bladder pain level was evaluated by a visual analogue scale in which 0 corresponded to "no pain" and 10 to "the worst pain imaginable". Patient's score was 10. Pelvic floor muscle examination was normal and neurologic examination did not reveal focal deficits. There was no lower extremity edema and there was no cervical, axillary or inguinal lymphadenopathy. Laboratory parameters were all normal (including PSA serum levels). Microscopic and bacteriological evaluation of urine and expressed prostatic secretion were negative as well as coproculture. Serological studies for viral hepatitis, HIV and syphilis were negative. A CT scan of the patient's abdomen showed only the presence of small retroperitoneal lymph nodes at the celiac tripod level and a unique $6 \mathrm{~mm}$ calcific sigmoidal diverticulum, confirmed by colonoscopy and unable to justify alone the abdominal pain. Cystoscopy revealed red areas involving the mucosa of the posterior and the left bladder walls. The prostatic urethra and the trigone appeared normal as did both ureteral orifices. Bladder capacity under anaesthesia was $380 \mathrm{~mL}$. After bladder hydrodistension the development of glomerulations did not occur. Transurethral biopsies revealed infiltrating eosinophils (Figure 1).

Postoperative course was unremarkable. Patient's postoperative symptoms decreased within 3 weeks taking corticosteroids (25 mg prednisone once-a-day) for 5 weeks. At 4 weeks postoperative he was given montelukast sodium. At 5 weeks postoperative pain score was 3 and bowel function improved. After discontinuing corticosteroids dysuria recurred (pain score 8) and itching, burning and hyperemia at the genital skin occurred. These symptoms temporarily resolved only at rest in a laying down position. In order to exclude parasitic origin of this dermatitis a specific enzyme-linked immunosorbent assay (ELISA) to detect antibodies against several parasites was slightly positive for Toxocara species. Montelukast sodium was discontinued and $1 \mathrm{mg}$ betamethasone dipropionate once-a-day was started together with albendazole $400 \mathrm{mg}$ by oral route 2 times per day with food for 5 days. Patient's symptoms improved and the pain score decreased up to 4 within 1 week.

\section{Figure 1.}

Histopathology from the bladder biopsy specimen demonstrating a nodular cystitis with abundant eosinophilic cells infiltration (hematoxylin-eosin stain).

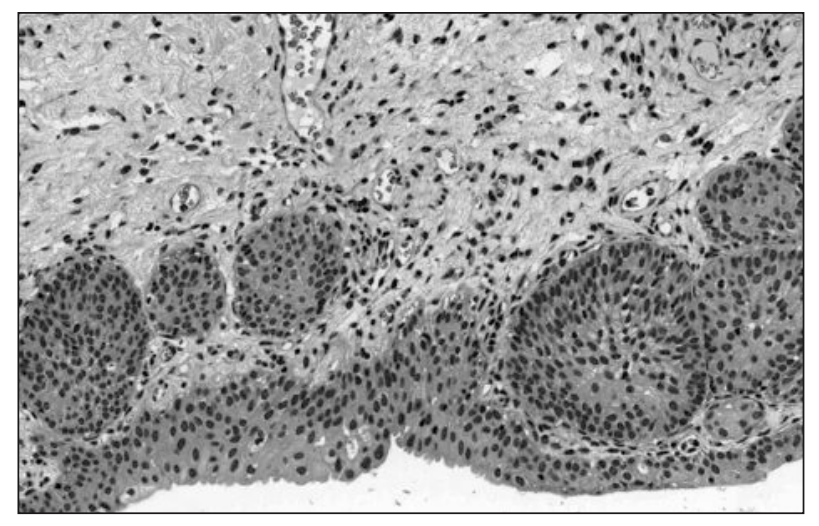

\section{Discussion}

The eosinophilic cystitis, although rare and mysterious, should be considered in adults with bowel and voiding symptoms associated to lower abdominal quadrant pain. Its management is a challenge. and should be tailored to each patient. Although leukotriene receptor antagonist (montelukast sodium) is useful in the treatment of eosinophilic cystitis in children, in adults this drug seems to be ineffective without corticosteroids (2). Steroidal anti-inflammatory drugs have been reported also to improve symptoms related to allergic response to parasitic infection such as Toxocariasis (3). In the present case a mild Toxocara infection with antibody titres only midly elevated (4) might be responsible for bladder and genital skin allergic response to the parasite also in the absence of serum eosinophilia.

\section{Conclusion}

The eosinophilic cystitis should be considered in adults with bowel and voiding symptoms associated to lower abdominal quadrant pain and its management should be tailored to each patient. In the present case a mild Toxocara infection with only midly elevated antibodies might be responsible for bladder and genital skin allergic response to the parasite also in the absence of serum eosinophilia.

\section{References}

1. Abrams P, Cardozo L, Fall M, et al. The standardisation of terminology in lower urinary tract function: Report from the Standardisation Sub-Committee of the International Continence Society. Urology. 2003; 61:37-49.

2. Sterret S, Morton J, Perry D, Donovan J. Eosinophilic cystitis: successful long-term treatment with montelukast sodium. Urology. 2006; 67:423.e19-423.e21.

3. Despommier D. Toxocariasis: clinical aspects, epidemiology, medical ecology, and molecular aspects. Clin Microbiol Rev. 2003; 16:265-272.

4. Leone $N$, Baronio $M$, Todros $L$, et al. Hepatic involvement in larva migrans of Toxocara canis: report of a case with pathological and radiological findings. Dig Liver Dis. 2006; 38:511-514.

\section{Correspondence}

Maria Angela Cerruto, MD

mariaangela.cerruto@univr.it

Carolina D'Elia, MD, FEBU (Corresponding Author)

karolinedelia@gmail.com

Walter Artibani, MD

walter.artibani@univr.it

Urology Clinic, Department of Surgery

University of Verona \& AOUI, p.le L Scuro 10 - 37134 Verona, Italy 